\title{
Social science research contributions to antimicrobial resistance: protocol for a scoping review
}

\author{
Abou Ali Vedadhir ${ }^{1,2}$, Carla Rodrigues ${ }^{1}$ (D) and Helen Lambert ${ }^{1 *}$ (D)
}

\begin{abstract}
Background: Antimicrobial resistance (AMR) is an escalating global health issue with complex and dynamic interdependencies, high uncertainty and decision stakes, multiple drivers and stakeholders with diverse values and interests, and various aspects and outcomes. Addressing and combating this critical global challenge requires the formation and establishment of an interdisciplinary research approach that goes beyond the biosciences principally concerned with antimicrobial resistance to include other relevant natural and social sciences. The objective of this study will be to review and map existing social science knowledge and literature relating to antimicrobial resistance.
\end{abstract}

Methods: The review team will undertake the scoping review using the Arksey and O'Malley methodological framework and also the Joanna Briggs Institute methods manual. Publications in English (from 1998 onwards) will be searched using several databases including PubMed/MEDLINE, Web of Science, Scopus, Anthropological Plus, Sociological Abstracts, International Bibliography of the Social Sciences (IBSS), PsycINFO and EconLit. Grey literature will also be searched (e.g. Google Scholar). Two reviewers will independently screen all citations, fulltext articles, and abstract data. Publication types will include original articles, editorials, commentaries, protocols, and books in the social science research literature on AMR. All study designs (quantitative, qualitative, and mixedmethods) will be included. A PRISMA Flow Diagram of search and study selection will be used to report final figures on included and excluded studies. To provide a descriptive summary of the literature, data will be collated, stored, and charted using Microsoft Excel software. The analysis will also involve identifying themes and gaps in the existing literature and summarizing, describing and displaying all pertinent information using thematic construction approaches including qualitative content analysis methods.

Discussion: This protocol describes a systematic method to identify, map, and synthesize social science research evidence on antimicrobial resistance. By mapping evidence and identifying potential knowledge gaps where further research is warranted, the resulting scoping review will provide useful insights for the design, implementation, and reorientation of future research agendas on AMR at multiple levels.

Systematic review registration: This protocol has been registered with the Open Science Framework (OSF): https://osf. io/hyaem.

Keywords: Social science research, Antimicrobial resistance (AMR), Scoping review

\footnotetext{
* Correspondence: h.lambert@bristol.ac.uk

'Department of Population Health Sciences, Bristol Medical School, University

of Bristol, Canynge Hall, 39 Whatley Road, Bristol BS8 2PS, UK

Full list of author information is available at the end of the article
}

(c) The Author(s). 2020 Open Access This article is distributed under the terms of the Creative Commons Attribution 4.0 International License (http://creativecommons.org/licenses/by/4.0/), which permits unrestricted use, distribution, and reproduction in any medium, provided you give appropriate credit to the original author(s) and the source, provide a link to the Creative Commons license, and indicate if changes were made. The Creative Commons Public Domain Dedication waiver (http://creativecommons.org/publicdomain/zero/1.0/) applies to the data made available in this article, unless otherwise stated. 


\section{Background}

Antimicrobial resistance (AMR) is widely regarded as one of the escalating global public health challenges of the 21st century [1]. AMR is an evolutionary process whereby microorganisms acquire the ability to withstand antimicrobial drugs, thus making treatment of infections ineffective and increasing the risk of resistant microorganisms spreading among people, animals, and the environment. The global spread of AMR may compromise our ability to treat existing and emerging common infectious diseases, as well as undermine many other improvements in health and sustainable development [2]. "Overuse" and "suboptimal use" of antimicrobials, including antibiotics in humans, farmed animals and the environment, mobility of human populations between regions and healthcare facilities, poor infection control, inadequate sanitary conditions and inappropriate food-handling are considered the main factors leading to the emergence and spread of AMR [3-7].

AMR is a growing, multifaceted public health problem that results in increased prolonged illness, disability and death for patients [4, 8]. Beyond all of these, AMR is widely considered to pose a threat to the future of humanity [9]. According to one estimate, if not curtailed, deaths per year linked with AMR could rise to 10 million by 2050 [10-12]. AMR also increases healthcare costs with lengthier stays in hospitals and a growing requirement for more intensive patient care [4, 12]. It has also been predicted that the mortality from infections subject to AMR could result in a reduction of $2 \%$ to $3.5 \%$ in global gross domestic product (GDP) in 2050, amounting to between 60 and 100 trillion US dollars worldwide [10, 11, 13]. According to the World Bank [14], people and economies in low- and middle-income countries (LMICs) will experience proportionately greater suffering than high-income countries (HICs) from reduced economic growth and global poverty caused by AMR. Health policymakers recognise that biomedical and life sciences approaches alone are insufficient to address critical global issues as significant and complex as AMR [15]. The adoption of a cross-sectoral 'one-health' or 'eco-health' approach has therefore been widely advocated [16-19]. Others have argued for a more 'overtly interdisciplinary' approach [20] or for an 'extended peer community' (engaged citizenry) approach to the issue [21-24], going beyond 'behaviour' [25, 26], 'resistance' [27], 'drugs and bugs' [28], 'academic tribes and territories' [29], and/or 'disciplinary epistemic cultures' [30, 31].

Together with calls for multidisciplinary and interdisciplinary research, AMR has been characterised as a social rather than a biological problem such that, it demands a social solution, one based on greater understanding, measurement, modelling, and ultimately (re)shaping the social, political, and economic environment in which resistance develops and antibiotics are used' [32]. There have been numerous calls to address the socio-cultural, economic, and political dimensions of AMR through greater involvement of social scientists in AMR research [25, 26, 33-45].

Yet, despite widespread agreement that social science research could provide vital insights into the sociocultural, economic, political, and organisational determinants and consequences of AMR [41], the social science literature on these issues is sparse and widely scattered [46]. This scoping review, therefore, aims to identify, categorise, summarize, synthesize and map existing knowledge, literature and evidence from published social science research addressing AMR. The available literature will be identified and synthesised in order to provide a map of current knowledge and to identify potential gaps where further social science research is warranted.

\section{Methods \\ Protocol design}

The methodological approach draws on a scoping review or scoping study framework. The general purpose of conducting scoping reviews is to identify and map the available evidence and to clarify key concepts/definitions in the literature. It is an ideal tool to determine the coverage of a body of literature on a given topic, or within a research area, and to give a clear indication of the volume of evidence or studies available, as well as a detailed overview of its focus [47-49]. A scoping review can also be used to provide a broad outline of a certain topic or a given field (such as AMR), to examine how research is conducted on a certain topic or field, to develop a "concept map" of a particular concept or approach in the literature-what it refers to, and what it encompasses - and to map evidence in relation to time, location, source (peer-reviewed or grey literature), and/ or origin (healthcare or other academic disciplines) [50]. In addition, scoping reviews can be useful to detect and analyse gaps in the existing body of knowledge and literature. They can, moreover, contribute to rapid evidence review in emerging fields or topics or to examine emerging evidence when it is still unclear what other, more specific, questions can be posed and valuably addressed by a more precise systematic review [49].

The present protocol has been registered within the Open Science Framework (registration: https://osf.io/ hyaem) and is being reported in accordance with the reporting guidance provided in the Preferred Reporting Items for Systematic Reviews and Meta-Analyses Protocols (PRISMA-P) statement [51, 52] (see checklist in Additional file 1). This study will be conducted as per the methodological framework developed by Arksey and 
O'Malley [47], the methodology outlined in the Joanna Briggs Institute Reviewers' Manual [48] and reported as per the PRISMA statement extension for scoping reviews (PRISMA-ScR) [53].

The stages of the study will comprise:

(1) Identification of the research questions and objectives

(2) Identification of relevant published studies and documents

(3) Selection of studies and documents

(4) Extracting and charting the evidence and data

(5) Collating, summarising and disseminating the results and identifying the implications of the study findings for policy, practice, and research on AMR.

This protocol was drafted using the PRISMA-ScR checklist and explanation, recently developed and revised by an international research team [53] with the aim of providing a rationale and an example of good reporting for each item of a systematic scoping review.

Oversight of protocol development, inclusion/exclusion criteria, and the review process will be provided by a multi-disciplinary advisory group including experts from medical anthropology, sociology, health economics, social psychology, health policy, and health services research.

\section{Stage one: identification of the research question(s) and objectives}

As mentioned above, the main aim of this scoping review is to identify, categorise, summarize, synthesize and map existing knowledge, literature and evidence from published social science research addressing AMR. More specifically, the review will be guided by the following research questions:

- What evidence or studies are available in the social science research literature that address AMR?

- What empirical, conceptual and theoretical elements constitute this body of literature?

- What knowledge and research gaps can be identified in the literature?

\section{Stage two: identification of relevant published studies/ literature}

Eligibility criteria To find and categorise all studies or evidence relevant to this review, the review team will search relevant databases (listed below) of published literature. Outputs will be included if they are: published from 1998 onwards, when the first major UK government review on AMR, "The Path of Least Resistance" was completed [54]; written and published in English only; addressing the phenomenon of evolving microbial resistance to existing antibiotic, antimicrobial or antifungal drugs; using social science approaches and/or methodologies [55], with social sciences defined as the disciplines included in the UK Economic and Social Research Council (UK ESRC)'s list [56]. Publication types will include original research articles, reviews, commentaries, short communications of findings, books and book chapters, protocol papers, theory/discussion papers, and editorials. Book reviews, wiki articles, blogs, websites, practice guidelines, leaflets and brochures, policy statements, reports on scientific meetings, and corporate literature and data will be excluded. All research designs (quantitative, qualitative, and mixed-methods studies) will be included. Studies and documents focused solely on clinical, biomedical, veterinary and microbiological aspects, mechanisms or processes of AMR will be excluded. Studies will also be included if they address human lives or life experiences, practices, organisations or structures associated with AMR and its drivers in non-human settings or subjects (e.g. veterinary or environmental aspects), but studies in animals, plants, or the natural environment will be excluded. As shown in Table 1, the Setting, Perspective, Intervention, Comparison, Evaluation (SPICE) framework $[57,58]$ is used to develop and outline inclusion/exclusion criteria and to frame the review questions. Reasons for inclusion of all relevant evidence will be documented at full-text review stage.

Information sources Potentially relevant studies, published in English between 1st January 1998 and 30th September 2019, will be searched in electronic databases and other relevant grey literature sources. The primary source of literature will be a structured search of major electronic databases and will include: Web of Science Core Collection; PubMed including Medline; Scopus; Sociological Abstracts; The International Bibliography of the Social Sciences (IBSS); Anthropological Plus; PsycINFO and EconLit. The secondary sources for searching grey or difficult to locate literature will include Google (Google Scholar and Google Books), Open Grey, ProQuest, and worldwidescience.org. Hand searches of the reference lists of included studies, reviews or other relevant documents, will be performed to identify additional relevant publications that may not be directly indexed in itemised sources. Content experts and authors who are prolific in the field will also be contacted. The literature searches will be designed and conducted by the review team in collaboration with two trained information specialists. The search will include a comprehensive range of terms and keywords related to social science disciplines, specific approaches and methodologies in social science research, and antibiotic, antimicrobial or drug resistance. For social science disciplines, as mentioned above, the search will adapt definitions from the UK ESRC's list [56]. A 
Table 1 Study details, characteristics, and results extraction instrument

\begin{tabular}{ll}
\hline Scoping review title: & Social science research contributions to antimicrobial resistance: a scoping review \\
Review objective/s: & To identify, categorise, summarize, synthesize and map out existing knowledge, \\
& literature and evidence on AMR from social sciences research \\
Review question/s: & $\begin{array}{l}\text { - What evidence or studies are available that address social, cultural, organizational, } \\
\text { political or economic dimensions of AMR? } \\
\text { - What empirical, conceptual and/or theoretical elements constitute this body of literature? } \\
\text { - What knowledge and research gaps can be identified? }\end{array}$ \\
Concepts (what*): & AMR, Social Sciences \\
Population (for whom*): & Humans (excluding studies conducted in animals and plants) \\
Core concept: & Social science research contributions to AMR \\
Language: & English \\
Date of publication: & January 1998-September 2019 \\
Data extraction: & Name (i.e., person extracting data) \\
& Date
\end{tabular}

Publication details

Author(s):

Title:

Type of publication/source (e.g. commentary/peer-reviewed journal)

Year and place of publication:

Aim(s)/research question(s):

Type of study and/or methodological approach (including data collection methods and analytical approach, if available)

Academic discipline/disciplinary approach (e.g. sociology, anthropology, economics):

Location (where*) (e.g. country/province; rural/urban; country income level):

Context (if applicable) (e.g. patients' home, primary/secondary/tertiary healthcare, pharmacies/ drug shops, farms, local/national/international policy):

Sample size (if applicable):

Year(s) of data collection:

Other results extracted from study or document content

Conceptual/theoretical framework or approach:

Domains addressed/focus of study

(e.g., prescribing, consuming or dispensing practices, social interactions including user-prescriber and/or professional—institutional interactions, formal/informal aspects, stockholders, contextual factors, drivers, costs and impacts, socio-cultural meanings, images and stigma, intervention development or evaluation, etc.);

Key findings that relate to the scoping review question(s) (*what result):

Comments on gaps, inconsistencies, biases and unmet needs in AMR research:

Reported AMR-related academic activities

(e.g., research and teaching programs, fellowships, funded projects; NGOs and networks; program and policy development, campaigns, advocacy, and knowledge exchange activities, regulation and delivery on AMR, etc.):

Other emerging information or themes (*what else):

*Components of the SPICE framework: Setting (where); Perspective/Population (for whom); Intervention/Phenomena of Interest (what); Comparison (what else); Evaluation (what result or how well)

draft search strategy for Web of Science is provided in Additional file 2. The final search results will be exported into EndNote X9 and all duplicates will be detected and then eliminated.

\section{Stage three: selection of studies and documents}

A two-stage selection process will be adopted consisting of an initial screening based on title and abstract only, followed by a full-text review of included items. All titles and abstracts retrieved in the search will be read, reviewed, and validated independently by two members of the research team. Documents not meeting the defined eligibility criteria will be excluded from full-text analysis.

Two reviewers will independently analyse the content of included full-text articles. The selected studies will be reread and re-evaluated by a third reviewer in cases of uncertainty or disagreement, and decisions about the eligibility of a publication resolved through discussion to reach consensus. 
Stage four: extracting and charting the evidence and data A standardised data abstraction template has been developed, adapted from the JBI Template extraction instrument [48]. The template will be used to extract data from full-text articles and other sources which meet the eligibility criteria (see Table 1). Two members of the research team will undertake data extraction and will review the completed template for each item. Any disagreements will be resolved through discussion and, when necessary, independent validation by the third reviewer (PI).

Piloting of the data abstraction template will be achieved through independent data extraction of three downloaded eligible articles purposively selected to be diverse in content by all members of the research team, followed by comparison and discussion to reach consensus on any modifications needed. At intervals during the data extraction and charting process, charted data will be compared and discussed to ensure consistency between reviewers and to enable iterative reflection on emerging themes and categories.

\section{Stage five: collating, summarising and disseminating the results and identifying the implications of the study findings for policy, practice and research on AMR}

A flow diagram, developed in line with PRISMA-ScR guidance [53], will be used to report the review searching and inclusion/exclusion pathway. To provide a descriptive summary of the literature, data will be collated, stored and charted using Microsoft Excel software. An empirical approach will be taken for descriptive characteristics (e.g. study type, method, location) but a narrative and flexible strategy will be employed in summarizing and synthesizing review findings on domains addressed and conceptual frameworks identified in the retrieved literature. To quantitatively describe characteristics of the included studies, key information or categories will be extracted from each source and presented using a variety of descriptive techniques including summary and frequency tables, graphs (e.g. Bubble Plot) and study matrices. Additionally, the analysis will involve identifying themes and gaps in the existing literature (both quantitative and qualitative) and summarising, describing and displaying all pertinent information using thematic construction approaches including content analysis methods. A narrative approach will be employed to describe themes that emerge from the extracted data; topics will be grouped by meaning and classified into coherent, relevant and clearly defined themes by two reviewers. The results will be compared and consolidated through accord between the two reviewers and the principal investigator (PI).

\section{Quality assessment and risk of bias}

The purpose of this scoping review is to recognise all the existing evidence or studies on the social science research literature that address AMR. As such, study quality or a formal risk of bias will not be assessed, nor will it be used as a basis for exclusion of studies. This is consistent with relevant guidance $[47,53]$.

\section{Discussion}

By systematically mapping existing social science research evidence on antimicrobial resistance and identifying potential knowledge gaps where further research is warranted, this scoping review will provide useful insights for the design, implementation, and orientation of future multi- and inter-disciplinary research on the global challenge of AMR at multiple levels: local, provincial, national, and global. The methodological process, key results, insights, and implications of this review may be of interest for diverse audiences and stakeholders inside and outside academia, including researchers, academics and policy-makers in a wide range of relevant fields beyond social sciences, given current acknowledgement of the relevance and value of social science research in addressing the complex global problem of AMR.

This review has several potential limitations. Although it acknowledges the need for 'one-health' or 'eco-health' interdisciplinary approaches to AMR, only publications relating to human populations, behaviours, and experiences (albeit including those which may influence environmental and veterinary aspects of AMR) will be included. Despite following well-recognised sources such as the UK ESRC and drawing on the expertise of researchers from a range of disciplines, determining what constitutes the content and scope of social sciences on the one hand and their contributions to AMR on the other is challenging. Increasing interdisciplinarity in the health sciences and the adoption of methods from the social sciences into a variety of other disciplines add to the difficulties of agreeing terminology and applicability and may result in the inadvertent exclusion of some relevant sources of evidence. Additionally, the lack of clarity in some studies regarding the disciplinary approach being taken may have implications for analytical categorisation of those contributions. The analysis of findings will take all these issues into consideration. The review team also acknowledges that social science research contributions to understanding AMR in societal context are not limited to published papers that directly address the problem of resistance (for instance, the substantial body of sociological and anthropological literature on medicines and pharmaceuticalisation is clearly relevant). A thorough approach to the literature, however, required narrowing down the selection criteria for this scoping review and these related literatures have been widely discussed previously. The review team believes this approach will improve the quality of the outcomes of this project. 
Ethical approval is not required as this scoping review solely entails secondary analysis of previously collected and publicly available publications and materials. However, the ethical precepts of copyright and intellectual property will be respected. During the review process, any amendments to the protocol that are deemed necessary by the review team will be recorded in the master protocol document and the reasons for the amendment noted on file; outputs reporting the review results will report any such amendments. The results of this review will be disseminated through peer-reviewed publications and presented at national and international conferences, targeting audiences interested or involved in research into AMR with a social science research approach.

\section{Supplementary information}

Supplementary information accompanies this paper at https://doi.org/10. 1186/s13643-020-1279-y.

Additional file 1. PRISMA-P 2015 Checklist.

Additional file 2. Search Strategy.

\section{Abbreviations}

AMR: Antimicrobial resistance; JBI: the Joanna Briggs Institute; PRISMAP: Preferred Reporting Items for Systematic Review and Meta-Analysis Protocols; PRISMA-SCR: The Preferred Reporting Items for Systematic reviews and Meta-Analyses extension for Scoping Reviews

\section{Acknowledgements}

The authors thank all members of the advisory group (Mustafa Al-Haboubi, Christie Cabral, Mishal Khan, Richard D. Smith and Carolyn Tarrant) for their valuable comments on an earlier draft of this protocol. We are grateful to Richard Kielb, James Webley and Sarah Herring (University of Bristol) and Fahimeh Ahmadian-Yazdi (University of Mysore) for their support and advice in identifying information sources and developing the search strategy for this review. Thanks also to the convenors, discussant, and participants in the 'Pharmaceuticals Panel' at the BSA Medical Sociology Group Annual Meeting in York, 11-13 September 2019 for their helpful comments and feedback on an earlier version of this protocol.

\section{Authors' contributions}

$\mathrm{HL}$ and AV conceived the study, outlined the proposal and are guarantors of the review. All authors have made a substantive intellectual contribution to developing this protocol, the review questions, and the review design. HL identified and validated the framework from which AV and CR developed and tested search terms. HL, AV, and CR jointly developed the search strategy and data extraction framework. All authors also drafted and edited the manuscript and approved the final version of the manuscript.

\section{Funding}

This work was supported by the Bristol AMR Research Strand (grant code: R100148-106) supported by Wellcome Trust ISSF and funded through the Elizabeth Blackwell Institute for Health (EBI) at the University of Bristol. http:// www.bristol.ac.uk/amr/bristol-amr-funded-projects-/

\section{Availability of data and materials}

All data generated or analysed during this study are included in this published article.

Ethics approval and consent to participate Not applicable

\section{Consent for publication}

Not applicable

\section{Competing interests}

The authors declare that they have no competing interests

\section{Author details}

${ }^{1}$ Department of Population Health Sciences, Bristol Medical School, University of Bristol, Canynge Hall, 39 Whatley Road, Bristol BS8 2PS, UK. ²Department of Anthropology, Faculty of Social Sciences, University of Tehran, Tehran 14117-13118, Iran.

Received: 8 November 2019 Accepted: 13 January 2020

Published online: 05 February 2020

\section{References}

1. Tacconelli E, Pezzani MD. Public health burden of antimicrobial resistance in Europe. Lancet Infect Dis. 2019;19(1):4-6.

2. WHO. Global action plan on antimicrobial resistance. Geneva: World Health Organization; 2015.

3. Hoffman SJ, Outterson K. Introduction: what will it take to address the global threat of Antibiotic Resistance? J Law Med Ethics. 2015;43(Suppl 3):6-11.

4. de Smalen AW, Ghorab H, Abd El Ghany M, et al. Refugees and antimicrobial resistance: A systematic review. Travel Med Infect Dis. 2017;5: 23-8.

5. Hay SI, Rao PC, Dolecek C, et al. Measuring and mapping the global burden of antimicrobial resistance. BMC Med. 2018;16:78.

6. Kamenshchikova A, Wolffs PFG, Hoebe CJ, et al. Complex narratives of health, stigma and control: antimicrobial resistance screening among nonhospitalized refugees. Soc Sci Med. 2018;212:43-9.

7. Catalán-Matamoros D, Pariente A, Elías-Pérez C. What we know about media communication on antibiotics and antimicrobial resistance: a systematic review of the scientific literature. Patient Educ Couns. 2019;102(8):1427-38.

8. Naylor NR, Atun R, Zhu N, et al. Estimating the burden of antimicrobial resistance: a systematic literature review. Antimicrob Resist Infect Control. 2018;7:58.

9. Smith, R. Does antimicrobial resistance pose "as great a threat to humanity's future" as climate change? BMJ Opin 2019. https://blogs.bmj.com/bmj/201 9/09/02/richard-smith-does-antimicrobial-resistance-pose-as-great-a-threatto-humanitys-future-as-climate-change/. accessed 20 Sept 2019.

10. O'Neill J. Tackling drug-resistant infections globally: final report and recommendations. The Review on Antimicrobial Resistance. London: HM Government and Welcome Trust; 2016.

11. de Kraker MEA, Stewardson AJ, Harbarth S. Will 10 million people die a year due to antimicrobial resistance by 2050? PLoS Med. 2016;13(11):e1002184.

12. Roope LSJ, Smith RD, Pouwels KB, et al. The challenge of antimicrobial resistance: What economics can contribute. Science. 2019;364(41):1-10.

13. Allcock SE, Young $\mathrm{H}$, Holmes MD, et al. Antimicrobial resistance in human populations: challenges and opportunities. Glob Health Epidemiol Genom. 2017;2:e4

14. World Bank. Drug-resistant infections: a threat to our economic future. Washington, DC: The World Bank; 2017.

15. Government of the UK. Tackling antimicrobial resistance 2019-2024: The UK's five-year national action plan. London: Department of Health and Social Care 2019. https://assets.publishing.service.gov.uk/government/ uploads/system/uploads/attachment_data/file/784894/UK_AMR_5_year_ national_action_plan.pdf . accessed 3 Sept 2019.

16. Charron DF. Ecohealth: Origins and Approach. In: Charron DF, editor. Ecohealth research in practice. innovative applications of an ecosystem approach to health. New York: Springer; 2012. p. 1-30.

17. Lang T, Rayner G. Ecological public health: the $21^{\text {st }}$ century's big idea? BMJ. 2012;345:e5466.

18. Roger F, Caron A, Morand S, et al. One Health and EcoHealth: the same wine in different bottles? Infect Ecol Epidemiol. 2016;6:30978.

19. Galway L, Parkes M, Allen D, et al. Building interdisciplinary research capacity: a key challenge for ecological approaches in public health. AIMS Public Health. 2016;3:389-406.

20. Holland D. Integrating knowledge through interdisciplinary research problems of theory and practice. London: Routledge; 2014.

21. Funtowicz SO, Ravetz JR. Science for the post-normal age. Futures. 1993; 25(7):739-55.

22. Funtowicz SO, Ravetz JR. Post-normal science (Chapter Three), in: International Society for Ecological Economics. ed. Online Encyclopedia of 
Ecological Economics 2003. http://isecoeco.org/pdf/pstnormsc.pdf. accessed 20 Aug 2019.

23. Ravetz JR. What is post-normal science? Futures. 1999;31(7):647-53.

24. Orderud Gl, Vogt RD, Tan $\mathrm{H}$, et al. Interdisciplinary research and transdisciplinary processes for environmental management under different socio-natural conditions. Int J Environ Stud. 2018:75(5):827-46.

25. Will CM. Editorial: Beyond behavior? Institutions, interactions and inequalities in the response to antimicrobial resistance. Sociol Health III. 2018;40(3):e1-9.

26. Will, C. The problem and the productivity of ignorance: public health campaigns on antibiotic stewardship. Sociological Review 2019 (in Press). http://sro.sussex.ac.uk/id/eprint/81753. accessed 10 Aug 2019.

27. EDCMA's Beyond Resistance Network (2019). Beyond resistance-our tiny non-humans: meet the microbes that shape our world (AMR in the Arts and Social Sciences). Edinburgh: Edinburgh Centre for Medical Anthropology (EDCMA). http://www.san.ed.ac.uk/edcma/edcma_networks/beyond_ resistance_network/festival_of_creative_learning_2019_beyond resistance_-_our_tiny_non-humans_meet_the_microbes_that_shape_our_ world. accessed 10 Aug 2019.

28. WHO. Tackling antimicrobial resistance (AMR) together. Working paper 5.0 Enhancing the focus on gender and equity. Geneva: World Health Organization; 2018

29. Becher T, Trowler PR. Academic tribes and territories-intellectual enquiry and the culture of disciplines. Buckingham: The Society for Research into Higher Education \& Open University Press; 2001.

30. Knorr-Cetina K. Epistemic cultures: how the sciences make knowledge. Cambridge: Harvard University Press; 1999.

31. Knorr-Cetina K. Culture in global knowledge societies: knowledge cultures and epistemic cultures. Interdiscip Sci Rev. 2007;32:361-75.

32. Smith R. Antimicrobial resistance is a social problem requiring a social solution. BMJ. 2015;350:h2682.

33. Radyowijati A, Haak H. Determinants of antimicrobial use in the developing world. Geneva: WHO; 2002

34. Chandler CIR, Hutchinson E, Hutchison C. Addressing antimicrobial resistance through social theory: an anthropologically oriented report. London: London School of Hygiene \& Tropical Medicine; 2016. p. 1-42.

35. Chandler, CIR, Hutchison C. Anthropology and antimicrobial resistance: Brief for ESRC AMR social science champion 2016. http://www.bristol.ac.uk/ media-library/sites/social-community-medicine/documents/social-scienceandamr/Anthropology\&AMR_02082016.pdf. accessed 22 July 2019.

36. Chandler CIR. Current accounts of antimicrobial resistance: stabilisation, individualisation and antibiotics as infrastructure: Palgrave Communications; 2019. p. 5.

37. Willis, LD, Chandler CIR. Anthropology's contribution to AMR control. AMR Control http://resistancecontrol.info/wp-content/uploads/2018/05/104-08chandler.pdf. accessed 22 July 2019.

38. British Dental Association. Social scientists needed to solve the problem of antibiotic overuse. Brit Dent J 2015;220 (15 Jan. 2016).

39. Lambert, H. (2015) Social scientists needed to solve the problem of antibiotic overuse. http://www.bristol.ac.uk/population-health-sciences/ projects/amr-champion/news/2015/social-scientists-needed-to-solve-theproblem-of-antibiotic-overuse.html . accessed 22 July 2019.

40. Lambert, $\mathrm{H}$. Championing antimicrobial resistance social science research. Impact 2019: 14-17. https:/www.ingentaconnect.com/content/sil/impact/2 018/00002018/00000004/art00006? crawler=true\&mimetype=application/pdf. accessed 22 July 2019.

41. Knight $\mathrm{G}$, Lambert $\mathrm{H}$, Feil $\mathrm{E}$, et al. The importance of cross-disciplinary research to combat antimicrobial resistance: introducing a new pop-up journal. X-AMR J Med Microbiol. 2018;67:1017-8.

42. Wood F. Antimicrobial resistance and medical sociology: research brief. Cardiff: ESRC AMR Research Champion/ University of Bristol; 2016.

43. Jensen CS, Nielsen SB, Fynbo L. Risking antimicrobial resistance: a collection of one-health studies of antibiotics and its social and health consequences. London: Palgrave Macmillan; 2019.

44. Brown N, Nettleton S. There is worse to come': the biopolitics of traumatism in antimicrobial resistance (AMR). Sociological Rev. 2017;65(3): 493-508.

45. Lambert $\mathrm{H}$, Chen M, Cabral C. Antimicrobial resistance, inflammatory responses: a comparative analysis of pathogenicities, knowledge hybrids and the semantics of antibiotic use. Palgrave Communications. 2019:5(85): $1-13$.
46. Frid-Nielsen SS, Rubin O, Baekkeskov E. The state of social science research on antimicrobial resistance. Soc Sci Med. 2019. https://doi.org/10.1016/j. socscimed.2019.112596.

47. Arksey H, O'Malley L. Scoping studies: towards a methodological framework. Int J Soc Res Methodology. 2005;8:19-32.

48. Peters MDJ, Godfrey C, Mclnerney P, et al. Scoping Reviews. In: Aromataris E, Munn Z. eds. Joanna Briggs Institute Reviewer's Manual. Adelaide: Joanna Briggs Institute, 2017. https://reviewersmanual.joannabriggs.org/ . accessed 26 July 2019.

49. Munn Z, Peters MDJ, Stern C, et al. Systematic review or scoping review? Guidance for authors when choosing between a systematic or scoping review approaches. BMC Med Res Methodol. 2018;818:143.

50. Anderson S, Allen P, Peckham S, et al. 'Asking the right questions: scoping studies in the commissioning of research on the organisation and delivery of health services. Health Res Policy Syst. 2008:6:7.

51. Moher D, Shamseer L, Clarke M, et al. Preferred Reporting Items for Systematic Review and Meta-Analysis Protocols (PRISMA-P)- 2015 statement. Syst Rev. 2015;4(1):1

52. Shamseer L, Moher D, Clarke M, et al. the PRISMA-P Group. Preferred Reporting Items for Systematic Review and Meta-Analysis Protocols (PRISMA-P) 2015: elaboration and explanation. BMJ. 2015;349:g7647.

53. Tricco AC, Lillie E, Zarin W, et al. PRISMA extension for scoping reviews (PRISMA-ScR Statement): Checklist and explanation. Ann Inter Med. 2018; 169:467-73.

54. Department of Health, UK (1998). The Path of Least Resistance: Main Report Standing Medical Advisory Committee (SMAC). Sub-group on Antimicrobial Resistance 1998. http://antibiotic-action.com/wp-content/uploads/2011/07/ Standing-MedicalAdvisory-Committee-The-path-of-least-resistance-1998.pdf accessed 25 Aug 2019

55. della Porta D, Keating M, editors. Approaches and methodologies in the social sciences: a pluralist perspective. Cambridge: Cambridge University Press; 2008.

56. ESRC. What is social science? UK: Economic and Social Research Counci 2019. https://escc.ukri.org/about-us/what-is-social-science/social-sciencedisciplines/. accessed 25 Aug 2019.

57. Stern C, Jordan Z, McArthur A. Developing the review question and inclusion criteria. Am J Nurs. 2014:114(4):53-6.

58. Booth A, Moore G, Flemming K, et al. Taking account of context in systematic reviews and guidelines considering a complexity perspective. BMJ Glob Health. 2019;4:e000840.

\section{Publisher's Note}

Springer Nature remains neutral with regard to jurisdictional claims in published maps and institutional affiliations.

\section{Ready to submit your research? Choose BMC and benefit from:}

- fast, convenient online submission

- thorough peer review by experienced researchers in your field

- rapid publication on acceptance

- support for research data, including large and complex data types

- gold Open Access which fosters wider collaboration and increased citations

- maximum visibility for your research: over $100 \mathrm{M}$ website views per year

At $\mathrm{BMC}$, research is always in progress.

Learn more biomedcentral.com/submissions 